\title{
Frequency-resolved optical gating with electro-optic sampling
}

\author{
Takao Fuji ${ }^{1, a}$, Yutaka Nomura ${ }^{1}$, Hideto Shirai ${ }^{2}$, and Noriaki Tsurumachi ${ }^{2}$ \\ 1 Institute for Molecular Science, 38 Nishigonaka, Myodaiji, Okazaki 444-8585, Japan \\ 2 Faculty of Engineering, Kagawa University, 2217-20 Hayashi-cho, Takamatsu, 761-0396, Japan
}

\begin{abstract}
We have demonstrated a new pulse characterization technique, cross-correlation frequency-resolved optical gating with electro-optic sampling. Sub-single-cycle mid-infrared pulses were characterized with the absolute carrier-envelope phase values by using the method.
\end{abstract}

Frequency-resolved optical gating (FROG) [1] is an important general technique to determine the intensity and phase evolution of an arbitrary ultrashort pulse. However, determination of its carrierenvelope phase (CEP) [2] was not possible with the method. On the other hand, electro-optic sampling (EOS) [3] is a method to obtain full information of the electric field including the absolute value of the CEP. Since the upper limit of the detectable frequency of the technique is the inverse of the pulse duration of the reference pulse, EOS has been used for characterization of the ultrashort pulses in rather low frequency region, specifically, from terahertz to mid-infrared region [4-6].

In this contribution, we propose a new pulse characterization scheme which is a combination of FROG and EOS. The method enables us to characterize an ultrashort pulse with the information of the absolute CEP value by using a reference pulse whose pulse duration is much longer than the period of the carrier-wave of the target pulse.

The time-averaged intensity of the delayed superposition of the fields of the reference pulse $\left(E_{\mathrm{ref}}(t-\right.$ $\tau))$ and the difference frequency between the reference and test pulses $\left(E_{\text {test }}(t)\right)$ is written as follows,

$$
\begin{aligned}
\langle| E_{\mathrm{ref}}(t-\tau) & \left.+\left.\alpha E_{\mathrm{ref}}(t-\tau) E_{\mathrm{test}}^{*}(t)\right|^{2}\right\rangle \\
= & \left\langle\left|E_{\mathrm{ref}}(t-\tau)\right|^{2}\right\rangle+\left\langle\left|\alpha E_{\mathrm{ref}}(t-\tau) E_{\mathrm{test}}^{*}(t)\right|^{2}\right\rangle+\left\langle 2 \mathfrak{R}\left\{\alpha E_{\mathrm{ref}}(t-\tau) E_{\mathrm{ref}}^{*}(t-\tau) E_{\mathrm{test}}(t)\right\}\right\rangle,
\end{aligned}
$$

where \langle\rangle denotes time average and $\alpha$ is a complex constant proportional to the nonlinear susceptibility of the difference frequency generation assumed as an instantaneous process. The first term is independent of the delay time. The second term is the intensity cross-correlation signal between the test pulse and the reference pulse. The spectrally resolved second term corresponds to a cross-correlation FROG signal (XFROG) [7]. The third term is the interference term, i.e., the EOS signal. If it is possible to assume $E_{\text {ref }}(t-\tau) E_{\text {ref }}^{*}(t-\tau) \equiv I_{\text {ref }}(t-\tau)$ as a delta function, the term becomes $E_{\text {test }}(\tau)$, which provides the complete information of the electric field of the test pulse. Otherwise, the term is spectrally filtered with the Fourier transform of $I_{\text {ref }}(t)$.

If we simultaneously measure the XFROG and EOS signals, i.e., the second and the third terms in eq. (1), the absolute value of the CEP obtained with EOS can be used for determining the CEP of the characterized pulse with XFROG. Thus, this technique permits us to completely retrieve an electric field which has too high frequency components to detect with EOS. In other words, the technique dramatically relaxes the requirement of the reference pulse for the full characterization of the target electric field.

\footnotetext{
a e-mail: fuji@ims.ac.jp
}

This is an Open Access article distributed under the terms of the Creative Commons Attribution License 2.0, which permits unrestricted use, distribution, and reproduction in any medium, provided the original work is properly cited. 

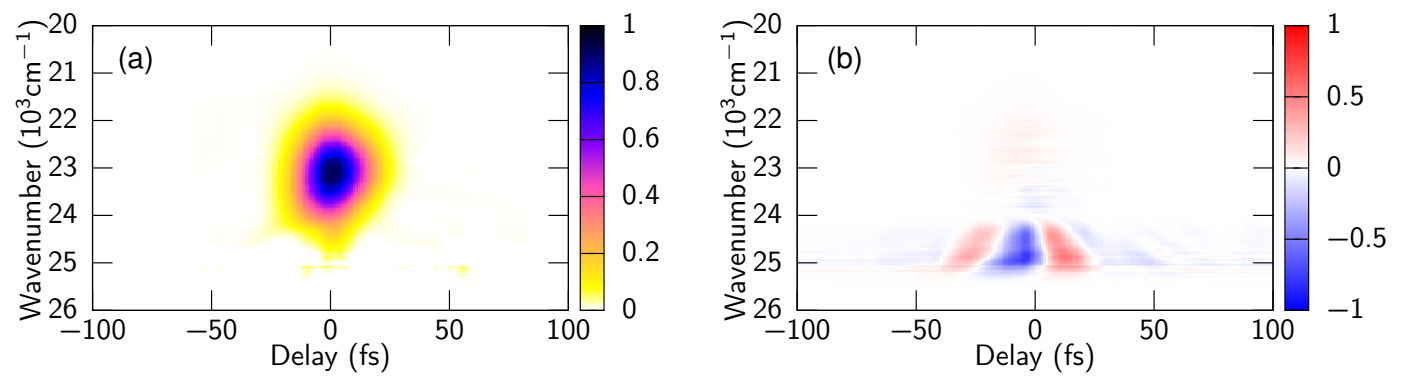

Fig. 1. Delay dependence of (a) summation and (b) difference of the two spectra described in the text.
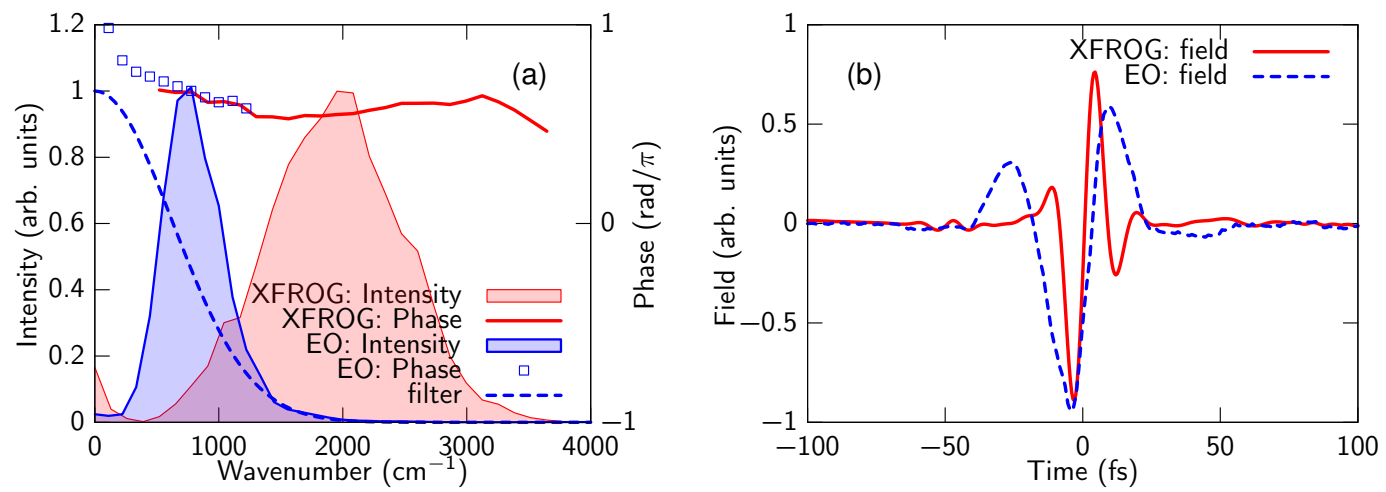

Fig. 2. (a) The retrieved intensity and phase in frequency-domain obtained from the XFROG (red) and EOS (blue). The dashed curve shows the filtering function for the EOS, Fourier transform of $E_{1}^{2}(t)$. The FROG error was $0.28 \%$ on a $256 \times 256$ grid. (b) The solid curve shows reconstructed electric field with the method described in the text. The dashed curve is obtained with integrating the difference spectrum in Fig. 1(b) along the wavelength axis.

Experimental demonstration of the scheme was realized with the system described as follows. We generated phase-stable sub-single-cycle pulses by using four-wave mixing of the fundamental and the second harmonic of Ti:sapphire amplifier (Femtopower compactPro, FEMTOLASERS) output through filamentation in argon, which is basically the same generation scheme as that reported in Ref. [8]. The generated mid-infrared pulse $\left(E_{\text {test }}(t), 150 \mathrm{~nJ}\right)$ was coupled with a small portion of the fundamental pulse $\left(E_{1}(t-\tau), 2 \mu \mathrm{J}, 25 \mathrm{fs}\right)$ with a delay time $\tau$ through a mirror with a hole. The combined beam was focused into argon again with a parabolic mirror $(f=150 \mathrm{~mm})$ and generated a four-wave difference frequency mixing $(\mathrm{FWM})$ signal, $E_{1}^{2}(t-\tau) E_{\text {test }}^{*}(t)$. Right after the focus, a thin BBO crystal $\left(\theta=29^{\circ}\right.$, Type $\left.1, t=50 \mu \mathrm{m}\right)$ was placed and generated the second harmonic $\left(E_{2}(t-\tau)=\right.$ $\left.E_{1}^{2}(t-\tau)\right)$ of the fundamental pulse. The polarization of the second harmonic was perpendicular to the FWM signal. The second harmonic and the FWM signal were mixed by a quarter wave plate and separated into two beams with a Wollaston prism. The intensities of the beams are basically explained as eq. (1) by substituting the square of the fundamental field, $E_{1}^{2}(t)$, into a reference field, $E_{\text {ref }}(t)$. Only difference between the two signals after the prism is the sign of the third term. The usual strategy at EOS is taking a difference between the signals of the two beams with a two channel detector, and measure only the third term of eq. (1) by using ellipsometry technique[9]. In our experiment, we measured the spectra of the two signals at the same time by using a imaging spectrometer with scanning the delay time $\tau$. Subtracting the spectrum contributed from the first term as a background, the summation of the two spectra reflects the spectra of the second term, the XFROG signal, and the difference between the two spectra reflects the spectra of the third term, the EOS signal. 
One example of the data set is shown in Fig. 1. Figure 1(a) and (b) show measured traces of summation and difference spectra, respectively. The retrieved intensity and phase in frequency-domain from Fig. 1(a) through XFROG retrieval algorithm are shown in Fig. 2(a). The signal in Fig. 1(b) was integrated along the wavelengths, then we obtained the electric field shown as the dashed curve in Fig. 2(b). The intensity and phase of the electric field in frequency-domain obtained with its Fourier transform are shown in Fig. 2(a). It is clear that the spectrum (the thick filled curve) is a result of filtering the original spectrum (the thin filled curve) through the Fourier transform of $\left|E_{1}^{2}(t)\right|^{2}$ (the dashed curve). Afterwards, an offset value was applied to the spectral phase obtained with the XFROG to match the both spectral phases in the overlapped region (from $500 \mathrm{~cm}^{-1}$ to $1500 \mathrm{~cm}^{-1}$ ). Finally, we retrieved the electric field of the generated mid-infrared pulse with the offset phase value as the solid curve in Fig. 2(b). Note that the slopes of the phases in the overlapped region $\left(500-1500 \mathrm{~cm}^{-1}\right)$ are well matched with each other, which means that group delays from the two independent measurements are matched with each other. The fact strongly supports the consistency of the data sets.

We controlled the CEP of the mid-infrared pulse by changing the delay between the fundamental and the second harmonic for the mid-infrared pulse generation with a feedback loop translation stage. The CEP changed with a period of $\sim 400 \mathrm{~nm}$, which is similar situation as Refs [10] and [11].

In conclusion, we described a new concept to characterize an ultrashort pulse with the absolute value of the CEP. We have demonstrated the method for characterizing phase-stable sub-single cycle mid-infrared pulses. In principle, the concept can be applied for any wavelength regions, then it is suitable for characterization of phase-stable single-cycle pulses in other wavelength regions $[12,13]$.

\section{References}

1. R. Trebino, Frequency-Resolved Optical Gating: The measurement of Ultrashort Laser Pulses (Kluwer Academic Publishers, 2000)

2. S.T. Cundiff, J. Phys. D 35, 43 (2002)

3. Q. Wu, X.C. Zhang, Appl. Phys. Lett. 67, 3523 (1995)

4. R. Huber, A. Brodschelm, F. Tauser, A. Leitenstorfer, Appl. Phys. Lett. 76, 3191 (2000)

5. A. Sell, R. Scheu, A. Leitenstorfer, R. Huber, Appl. Phys. Lett. 93, 251107 (2008)

6. I. Katayama, R. Akai, M. Bito, H. Shimosato, K. Miyamoto, H. Ito, M. Ashida, Appl. Phys. Lett. 97, 021105 (2010)

7. S. Linden, H. Giessen, J. Kuhl, Phys. Status Solidi B 206, 119 (1998)

8. T. Fuji, T. Suzuki, Opt. Lett. 32, 3330 (2007)

9. M. Born, E. Wolf, Principles of Optics (Wiley, New York, 1984)

10. C. Manzoni, M. Först, H. Ehrke, A. Cavalleri, Opt. Lett. 35, 757 (2010)

11. A. Thai, M. Hemmer, P.K. Bates, O. Chalus, J. Biegert, Opt. Lett. 36, 3918 (2011)

12. G. Krauss, S. Lohss, T. Hanke, A. Sell, S. Eggert, R. Huber, A. Leitenstorfer, Nature Photonics 4, $33(2010)$

13. S.W. Huang, G. Cirmi, J. Moses, K.H. Hong, S. Bhardwaj, J.R. Birge, L.J. Chen, E. Li, B.J. Eggleton, G. Cerullo et al., Nature Photonics 5, 475 (2011) 